\title{
Deep Brain Stimulation to Reward Circuitry Alleviates Anhedonia in Refractory Major Depression
}

\author{
Thomas E Schlaepfer*,1,2, Michael X Cohen ${ }^{3,4}$, Caroline Frick', Markus Kosel', Daniela Brodesser', \\ Nikolai Axmacher ${ }^{3}$, Alexius Young Joe ${ }^{5}$, Martina Kreft', Doris Lenartz ${ }^{6}$ and Volker Sturm ${ }^{6}$ \\ 'Department of Psychiatry and Psychotherapy, University Hospital, Bonn, Germany; ${ }^{2}$ Departments of Psychiatry and Mental Health, The Johns \\ Hopkins University, Baltimore, MD, USA; ${ }^{3}$ Department of Epileptology, University Hospital, Bonn, Germany; ${ }^{4}$ Department of Psychology, \\ University of California, Davis, CA, USA; ${ }^{5}$ Department of Nuclear Medicine, University Hospital, Bonn, Germany; ${ }^{6}$ Department of Functional \\ Neurosurgery, University Hospital, Cologne, Germany
}

Deep brain stimulation (DBS) to different sites allows interfering with dysfunctional network function implicated in major depression. Because a prominent clinical feature of depression is anhedonia-the inability to experience pleasure from previously pleasurable activities - and because there is clear evidence of dysfunctions of the reward system in depression, DBS to the nucleus accumbens might offer a new possibility to target depressive symptomatology in otherwise treatment-resistant depression. Three patients suffering from extremely resistant forms of depression, who did not respond to pharmacotherapy, psychotherapy, and electroconvulsive therapy, were implanted with bilateral DBS electrodes in the nucleus accumbens. Stimulation parameters were modified in a double-blind manner, and clinical ratings were assessed at each modification. Additionally, brain metabolism was assessed I week before and I week after stimulation onset. Clinical ratings improved in all three patients when the stimulator was on, and worsened in all three patients when the stimulator was turned off. Effects were observable immediately, and no side effects occurred in any of the patients. Using FDG-PET, significant changes in brain metabolism as a function of the stimulation in fronto-striatal networks were observed. No unwanted effects of DBS other than those directly related to the surgical procedure (eg pain at sites of implantation) were observed. Dysfunctions of the reward system - in which the nucleus accumbens is a key structure - are implicated in the neurobiology of major depression and might be responsible for impaired reward processing, as evidenced by the symptom of anhedonia. These preliminary findings suggest that DBS to the nucleus accumbens might be a hypothesis-guided approach for refractory major depression.

Neuropsychopharmacology (2008) 33, 368-377; doi:I0.1038/sj.npp. I 30 I408; published online I I April 2007

Keywords: deep brain stimulation; major depression; anhedonia; nucleus accumbens; brain stimulation; Cg25

\section{INTRODUCTION}

Traditional methods of alleviating depression largely stem from serendipitous observations of antidepressant effects of substances such as iproniazid (originally developed as a treatment for tuberculosis) or imipramine (originally developed as a treatment for schizophrenia). In particular, increasing levels of monoamine neurotransmitters in the synaptic cleft are associated with improvements of depressive symptoms. This insight led to a more targeted drug discovery process, resulting in drugs with fewer side effects, such as SSRIs. These medication treatments, in conjunction with certain methods of psychotherapy and electroconvulsive therapy, are effective at alleviating depressive sympto-

*Correspondence: Dr TE Schlaepfer, Department of Psychiatry, University Hospital, Sigmund-Freud-Strasse 25, 53105 Bonn, Germany, Tel: + 49228287 |47|5, Fax: + 49228287 |5025,

E-mail: schlaepf@jhmi.edu

Received 8 August 2006; revised 26 February 2007; accepted 27 February 2007 matology in most patients (Andrews and Nemeroff, 1994; Mann, 2005). However, these treatments do not work for all patients. A sizable minority of patients does not respond. Indeed, twelve percent of patients suffering from major depression have a poor outcome even after 5 years of treatment (Keller et al, 1992). Patients who do not respond to any known treatment combination including electroconvulsive therapy are thus referred to as 'treatmentresistant' patients, and typically have little hope of recovering.

Psychotropic drugs work by altering neurochemistry to a large extent in widespread regions of the brain, many of which may be unrelated to depression. We believe that more focused, targeted treatment approaches that modulate specific networks in the brain will prove a more effective approach to help treatment-resistant patients. In other words, whereas existing depression treatments approach this disease as a general brain dysfunction, a more complete and appropriate treatment will arise from thinking of depression as a dysfunction of specific brain networks that mediate mood and reward signals, in particular, the 
cortical-limbic-thalamic-striatal network (Mayberg, 2002). This conceptualization leads to novel ideas about targeted neuromodulatory treatments.

Recent advances in stereotaxic neurosurgical methods have provided a novel and promising technique for alleviating depression in treatment-resistant patients. This technique is deep brain stimulation (DBS), and refers to the stereotaxic placement of unilateral or bilateral electrodes in target brain regions connected to a permanently implanted neurostimulator, which electrically stimulates that brain region (Schlaepfer and Lieb, 2005). Today, DBS is widely used as a treatment for symptoms of Parkinson's disease (Ghika et al, 1998; Greenberg and Rezai, 2003; McClelland et al, 2005). In these patients, electrodes are placed in the subthalamic nucleus or the globus pallidus internus, and provide immediate recovery to otherwise debilitating motor symptoms. In psychiatric disorders, DBS is used in patients with refractory obsessive-compulsive and Tourette's disorders, and preliminary results suggest that DBS is an effective treatment (Abelson et al, 2005; Flaherty et al, 2005; Greenberg et al, 2003; Gross, 2004; Houeto et al, 2005) and stimulation of the nucleus accumbens for this indication has been proposed by one of the authors (Sturm et al, 2003). One group has recently reported the use of DBS in patients with major depression (Mayberg et al, 2005). In this study, Mayberg and colleagues implanted electrodes in white matter close to subgenual cingulate in Brodmann area 25 (Cg25). Results from this study are preliminary but promising, and open new possibilities for alleviating depression in otherwise treatment-resistant patients. The authors chose $\operatorname{Cg} 25$ on the basis of previous findings that this region is implicated in acute induced sadness, is metabolically overactive in treatment-resistant depression, and that clinical improvement after pharmacotherapy and psychotherapy is correlated with decreases in its metabolic activity (Auer et al, 2000; Mayberg et al, 1999; Mirza et al, 2004; Seminowicz et al, 2004).

We believe that DBS to the ventral striatum, and in particular, the nucleus accumbens (Figure 1), will be a promising and efficacious treatment of severe depression. Our hypothesis is based on three lines of reasoning: (1) the ventral striatum is heavily implicated in both normal and abnormal reward processes, (2) the nucleus accumbens acts as a 'motivation gateway' between limbic systems involved in emotion and systems involved in motor control, and (3) the ventral striatum is uniquely located to modulate activity in other regions of the brain. These arguments are described in detail below.

First, the ventral striatum, and in particular the nucleus accumbens, is a central region for processing reward and pleasure information. Increases in nucleus accumbens neuron activity and dopamine release are observed during expectations and experience of rewards (Adinoff, 2004; de la Fuente-Fernandez et al, 2002; Doyon et al, 2005; Schultz, 2004). Converging evidence of the accumbens' role in reward processing comes from neuroimaging studies, which show increases in ventral striatal activity associated with euphoric responses to dextroamphetamine (Drevets et al, 2001), cocaine-induced euphoria (Breiter et al, 1997), monetary reward (Cohen et al, 2005; Knutson et al, 2001a; O'Doherty et al, 2001), pleasurable responses to music (Blood and Zatorre, 2001), and viewing attractive faces
(Aharon et al, 2001). Recent evidence demonstrates that the ventral striatum exhibits abnormal activity following administration of dextroamphetamine in patients with major depression, compared with activity observed in healthy control subjects (Tremblay et al, 2005). Furthermore, data exploring a specific role of the nucleus accumbens within the limbic system demonstrate that the reward system is dysfunctional in mice submitted to social defeat stress, a dysfunction which is reset by chronic administration of an antidepressant (Berton et al, 2006). Together, these findings demonstrate that the nucleus accumbens is a critical center for the experience of reward and pleasure, and that this region is dysfunctional in patients who suffer from depression.

Second, the nucleus accumbens acts as a gateway to transmit, and therefore enhance or degrade, information from emotion centers of the brain to motor control regions of the brain. For example, depleting dopamine from the shell region of the nucleus accumbens in rats severely impairs their ability to engage in reward-seeking behaviors (Ito et al, 2004). Human neuroimaging studies have shown that the ventral striatum is very active during rewardseeking behaviors (Juckel et al, 2006; Knutson et al, 2001b, 2003), and this activation is reduced in certain clinical populations; for example, patients with schizophrenia (Juckel et al, 2006). Thus, the nucleus accumbens mediates motivational behavior related to obtaining rewards. This is particularly relevant to the treatment of depression because anhedonia, which can be conceptualized as lack of rewardmotivated behavior, is one of the key defining symptoms of the disorder.

Third, the ventral striatum is in a particularly unique position to modulate activity in many other regions of the brain. The nucleus accumbens receives projections from midbrain regions that produce dopamine such as the ventral tegmental area, from regions involved in emotion such as the amygdala, orbitofrontal cortex, and medial prefrontal cortex, from motor regions such as the dorsal caudate and globus pallidus, and from regions involved in memory such as the hippocampus (Nauta and Domesick, 1984). The accumbens in turn indirectly projects to cortical regions including Cg25 and medial prefrontal cortex, the ventral pallidum, the thalamus, amygdala, and hypothalamus (Jones and Mogenson, 1980; Kelley and Stinus, 1984; Mogenson et al, 1983). Many of these regions are also implicated in normal and abnormal emotion processing, especially the medial prefrontal cortex and Cg25, suggesting a network of tightly anatomically and functionally connected regions (Mayberg, 1997). These connections can be GABA-ergic (inhibitory) or glutamatergic (excitatory). Thus, stimulating the nucleus accumbens can modulate neural activity in other emotion and motivation centers of the brain. This is further evidenced by our PET data, described below.

Based on this reasoning, we included three patients with severe, treatment-resistant depression to take part in our study on the effects of DBS in the ventral striatum on depression. These patients did not respond to adequate traditional methods including combinations of psychotherapy, psychopharmacological drugs, and electroconvulsive therapy (see Methods). Electrodes were implanted bilaterally in the ventral striatum. Each electrode has four 


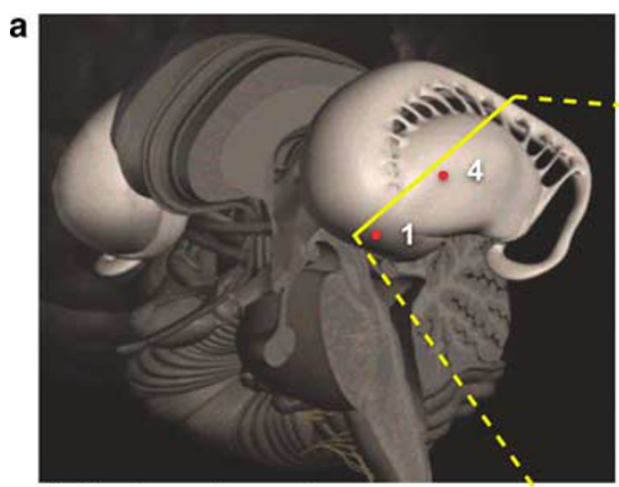

1 Nucleus Accumbens 2 Ventral Pallidum 3 Terminal Stria 4 Putamen 5 External Globus Pallidus 6 Internal Globus Pallidus
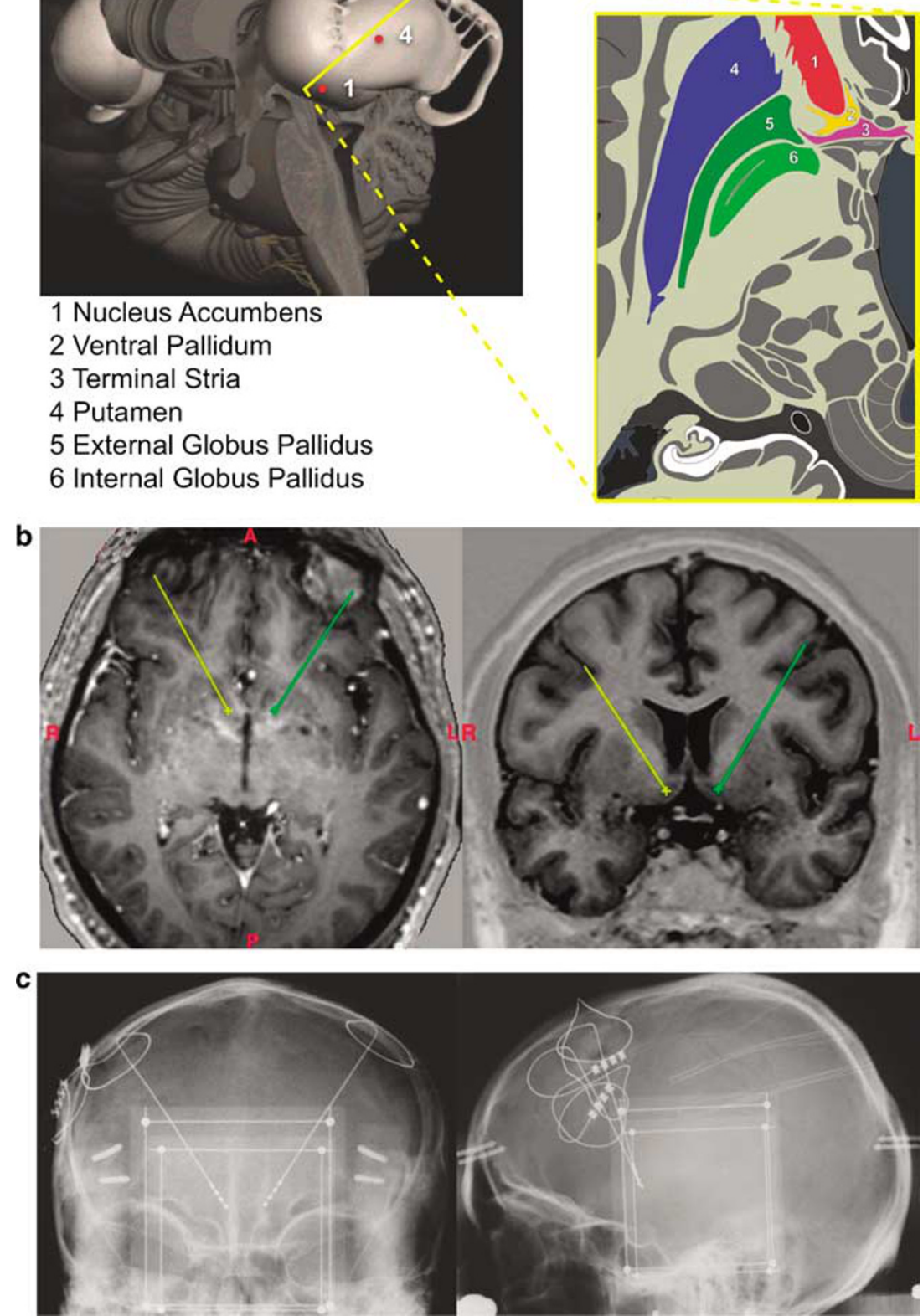

Figure I Location of nucleus accumbens and position of deep brain stimulation electrodes. (a) The topographical location of the nucleus accumbens in relation to other brain structures on a horizontal plane $3 \mathrm{~mm}$ below the AC-PC plane. (b) The location of the lowest contact of the stimulation electrode in a horizontal and coronal plane with projections of the left (green) and right (yellow) electrode path in the surgical planning stage. Stereotaxic coordinates are $1.5 \mathrm{~mm}$ rostral to the anterior edge of the anterior commissure, measured at the crossing point, $4 \mathrm{~mm}$ ventral and $7-8 \mathrm{~mm}$ lateral of the midline of the third ventricle. Burr holes were placed deep fronto laterally. (c) The actual location of the electrode leads in the post-operative control X-ray.

contacts: (1) the shell and (2) the core regions of the nucleus accumbens, and (3) the ventral and (4) the medial internal capsule (see Figure 2). Electrodes were connected to an automatic stimulator (ie not controllable by the patients), which was implanted subcutaneously over the breast muscle. We report here results of two experiments that demonstrate acute, amelioratory effects on depression as a result of DBS. In the first experiment, we methodically switched stimulation on or off in a double-blind manner over the course of several months and recorded, at each change, clinical ratings of depression. In the second experiment, we conducted PET scans before stimulation and 1 week after stimulation onset. Results from these two experiments demonstrate that DBS has an unequivocally beneficial effect on symptoms of depression, and suggest that these effects are the result of electrical stimulation of the Nucleus Accumbens, a key structure of the reward system. This is especially striking considering that these patients were not helped by any other treatment methods. 


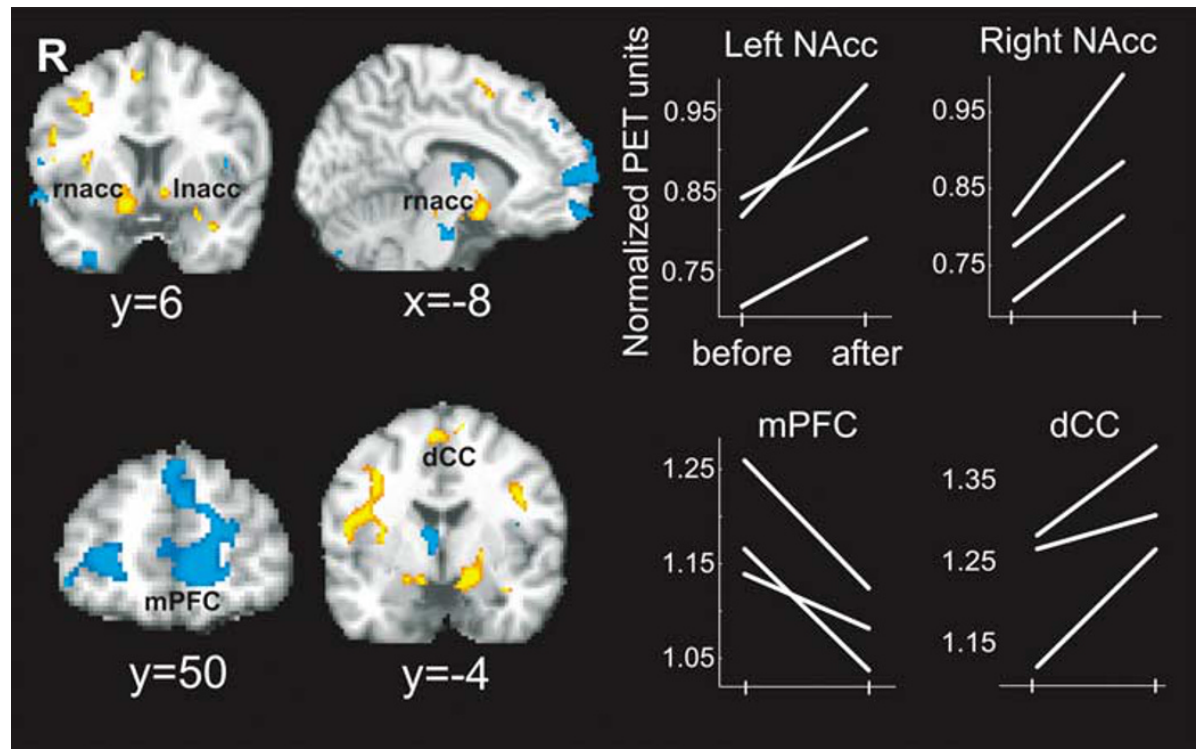

Figure 2 One week after onset of bilateral stimulation to the nucleus accumbens, metabolism in a distributed network of limbic and prefrontal brain regions was altered. Increases in metabolism following stimulation (shown in yellow) were observed in the nucleus accumbens, amygdala, dorsolateral, and dorsomedial prefrontal cortex. Decreases in metabolism (shown in blue) were observed in medial prefrontal cortex and caudate. Graphs on the right display normalized PET units from each subject from before and after implantation in the nucleus accumbens (NAcc), the medial prefrontal cortex (mPFC), and the dorsal cingulate cortex (dCC).

\section{METHODS}

We report here on first preliminary results obtained in the first three patients from a investigator initiated doubleblinded and placebo controlled study protocol aiming to assess putative effects of bilateral DBS to the nucleus accumbens on depression and quality of life in treatmentrefractory patients.

\section{Patients}

All patients had in common that they suffered from very treatment-refractory major depression; to be included in the study treatment trials with psychotherapy, pharmacotherapy and ECT had to have failed. From a time point 6 weeks before the study, patients were required to be on stable psychotropic medication, no other concomitant treatment was allowed; throughout the study period reported here medication was continued unchanged.

Patient 01 is a 66-year-old woman suffering from major depression since age 21 . The current depression episode was her sixth, and lasted for 17 months. During the current episode, the patient failed seven antidepressant medication trials, and augmentation with five different neuroleptics also failed. The patient did not respond to both an adequate trial of psychotherapy and 13 treatments of bilateral ECT. At study entry the patient was severely depressed, with a Hamilton Depression scale $\left(\mathrm{HDRS}_{24}\right.$ ) score of 38. At study entry she was treated with $90 \mathrm{mg}$ duloxetine, $1 \mathrm{mg}$ risperidone, $10 \mathrm{mg}$ diazepam, $75 \mathrm{mg} \mathrm{L}$-thyroxin, and $75 \mathrm{mg}$ melperone.

Patient 02 is a 37-year-old unemployed university graduate (economics). The current episode was his second, and began 9 years ago. Seventeen different antidepressant medication treatments have failed, as did augmentation with five neuroleptics and lithium. The patient had four courses of unsuccessful ECT treatments with at least 10 treatment sessions (twice unilateral, twice bilateral), and psychotherapy was tried, again to no avail. At study entry he was treated with $50 \mathrm{mg}$ quietapine and $75 \mathrm{mg}$ amitryptiline.

Patent 03 is the 37-year-old monozygotic twin brother of patient 02; unipolar major depression had developed in both patients in almost exactly the same way at the same time, he had the same professional education and current state as his brother. His current episode began 11 years ago, and this patient has been unsuccessfully treated with at least eight antidepressant medications, and augmentation with at least three neuroleptics and with lithium failed. The patient had two ECT treatment courses (one unilateral and one bilateral), comprising 10 and 15 treatment sessions, respectively, with no success, and psychotherapy also failed. Both brothers (patients 02 and 03) are economics graduates at the university level, but have never been able to work because of onset of their psychiatric illness. They were both living again with their parents in the same household. At study entry they were severely depressed (HDRS 24 score of 31 and 32). At study entry he was treated with $50 \mathrm{mg}$ quietapine, $150 \mathrm{mg}$ amitryptiline, and $75 \mathrm{mg}$ L-thyroxin.

\section{Ethical Considerations}

Written informed consent was obtained after all procedures had been explained fully. The ethics committees at the Universities Bonn and Cologne approved this study. In addition, and not stipulated by our ethics committee, an independent psychiatrist (ie not part of our research group) reviewed each patient and clinical data with respect to inclusion and exclusion criteria. 


\section{DBS Method}

DBS electrode implantation was performed stereotactically; standard Medtronic model 3387 leads were used. This lead has four electrodes over a length of $10.5 \mathrm{~mm}$, each spaced $1.5 \mathrm{~mm}$ apart. Intraoperative X-ray was used to verify the positioning of the two electrodes. Only the two lowest of the four contact sites (the lowest one placed in the shell of the nucleus accumbens, the other in its core) of each electrode were used for stimulation. Target-point and trajectory were defined using stereotaxic 3 Tesla MR imaging. DBS treatment was applied with permanent pulse-train stimulation (square-wave impulses of $90 \mu$ s pulse width, $145 \mathrm{~Hz}$ and amplitudes of $4 \mathrm{~V}$, electrodes negative against case), those stimulation parameters were chosen because of the extensive experience with neurostimulation for neurological disorders (Deuschl and Bain, 2002; Deuschl et al, 2006).

\section{Clinical Ratings}

At each study visit, we assessed each patient using over 20 clinical and neuropsychological measures. However, here we limit ourselves to reporting findings obtained from the Hamilton (1960) and Montgomery and Asberg (1979) depression rating scales because these are the most widely used and accepted measures of depression. Nonetheless, findings reported here were consistent across all measures of depression used.

\section{PET Imaging}

We acquired two FDG-PET scans from each patient, once 1 week before implantation and once 1 week after stimulation. Before the second FDG-PET examination, patients received stimulation at increasing voltages up to $4 \mathrm{~V}, 145 \mathrm{~Hz}$ during 7 days. Imaging was performed on a PET/CT scanner in 3-D mode (Biograph; Siemens Medical Solutions Inc.) with a $15.8-\mathrm{cm}$ axial field of view and an in-plane spatial resolution of $4.6 \mathrm{~mm}$, according to the procedure guidelines for brain imaging using ${ }^{18}$ FDG-PET of the European Association of Nuclear Medicine (Bartenstein et al, 2002). Blood glucose measured before tracer injection was $96 \pm 11 \mathrm{mg} / \mathrm{dl}$. The patients were positioned comfortably in a quiet, dimly lit room several minutes before FDG administration and during the uptake phase of FDG. Scans were performed $30 \mathrm{~min}$ after intravenous injection of $\sim 200 \mathrm{MBq}$ of ${ }^{18} \mathrm{FDG}$. The acquisition time was 20 min per position. Low-dose CT for attenuation correction was performed within $1 \mathrm{~min}$ before PET administration with the patient in the same position. The acquisition parameters for dual-detector helical CT were $130 \mathrm{kV}, 16 \mathrm{mAs}, 3-\mathrm{mm}$ slice thickness, and a pitch of 1.2.

Sixty-four slices were acquired $(\mathrm{FOV}=332.8,256 \times 256$ Matrix, voxel size $=1.3 \times 1.3 \times 2.43 \mathrm{~mm}$ ). Images were normalized into standard space, resampled to $2 \mathrm{~mm}$ isotropic voxels, smoothed using a $10 \mathrm{~mm}$ FMHW gaussian kernel, and scaled to correct for whole-brain mean differences. Differences in PET values at each voxel between 'before' and 'after' images were assessed using a general linear model in SPM2 with time as the covariate of interest. Non-sphericity due to inherent spatial autocorrelation was corrected for. The resulting t-map was thresholded at $p<0.05$ (two-tailed), and a cluster threshold of 50 voxels overlaid on top of a single subject's T1-weighted MRI scan.

\section{RESULTS}

Subjective effects were assessed using the morphine-benzedrine group (scores range 0-16) subscale of the Addiction research center inventory, which reflects feelings of euphoria and well-being (Jasinski et al, 1984), scores were 0 for all patients for both conditions (stimulation 'on' and 'off). There were no 'liking' (Kornetsky, 2004) effects during stimulation, in contrast to findings reported by Heath, who observed that human self-stimulation by electrodes in subcortical structures induced extreme rewarding effects (Heath, 1972). Indeed, none of our patients was able to determine whether DBS stimulation was on or off at any time.

\section{Immediate Clinical Effects of Stimulation}

Almost immediately (60s) after switching the stimulation on, one patient was unable to identify any changes, but spontaneously reported that he realized that he was in Cologne, that he never visited the famous Cologne Cathedral, and he planned on doing this in the immediate future, which he indeed did the day following the operation. Asked about depressive symptomatology, he did not report any acute subjective changes. A second patient's immediate (60 s) reaction to stimulation was quite similar; she did not report any acute changes in depressive symptomatology but spontaneously mentioned that she wished to take up bowling again (a favorite pastime of hers 12 years ago, before onset of her depression). She noted, 'This would be quite pleasurable.' These immediate and unprompted behavioral responses demonstrate a sharp increase in exploratory motivation, consistent with the accumbens' role in reward-seeking behaviors. This is especially noteworthy given these patients' severe lack of motivation during their long depressive episode.

\section{Longer Term Clinical Effects of Stimulation}

We manipulated the voltage of the stimulation in all three patients in a double-blind manner. Voltage was varied between 0 (eg no stimulation) and $4 \mathrm{~V}(5 \mathrm{~V}$ in the case of patient 2) in $1-\mathrm{V}$ steps. At each change in voltage setting, clinical ratings including the $\mathrm{HDRS}_{24}$ and MADRS were taken. Baseline $\mathrm{HRSD}_{24}$ score was $33.7( \pm 3.8)$ and baseline MADRS score was $35.7( \pm 2.9)$, indicating a severe level of depression. The scores dropped significantly to $19.7( \pm 6.7)$ and $24.7( \pm 6.7)$ after 1 week of deep brain stimulation (titration period) $(t(2)=4.68 ; p=0.02$, and $t(2)=4.66$; $p=0.02)$. After the first week of double-blind stimulation, the scores dropped to $24.7( \pm 3)(t(2)=2.45 ; p=0.07)$ and $26( \pm 3)(t(2)=9.67 ; p=0.04)$, respectively. After the first week without stimulation (double-blind), the scores increased again to $29.3( \pm 5.5)$ and $33.3( \pm 9.71)$. These ratings in the off-stimulation phase did not differ from baseline $(t(2)=0.81 ; p=0.25$ and $t(2)=0.33 ; p=0.39)$. In addition, we analyzed ratings for single items of both the $\mathrm{HDRS}_{24}$ and the MADRS scale thought to capture aspects of anhedonia, such as 'work and activities' and 'genital symptoms' in the $\mathrm{HDRS}_{24}$ and 'apparent sadness', 'concentration difficulties', 
'lassitude', and 'inability to feel' in the MADRS. For none of the items significant changes could be observed (in contrast to the clear and acute clinical changes in anhedonia observed), possibly pointing to limitations of these depression rating scales in assessing the full picture of depressive states. Figure 3 displays clinical ratings as a function of time, and whether stimulation was on or off (gray shading bars indicate that stimulation was on).

Clinical ratings improved in all three patients when the stimulator was on, and worsened in all three patients when the stimulator was turned off. These effects were immediate and bi-directional (eg when the stimulator was turned off, depression ratings immediately worsened, and when the stimulator was turned on, depression ratings immediately improved). The longer term effect on patient 3 is less robust, although despite this immediate two-way effects of stimulation on clinical rating scores could be observed.

We quantified the effects of stimulation on clinical ratings by correlating the ratings (re-coded as percent change from pre-implantation levels to be able to compare across patients and measures) with stimulation parameters. We found a negative correlation (ie increased stimulation led to decreased depression ratings) in all patients for the HDRS 24 $(r=-0.73,-0.44,-0.40)$ and MADRS $(r=-0.85,-0.36$, $-0.41)$ scores. These correlations were significant across patients (one-sample $t$-test; Hamilton: $t_{2}=-5.04, p<0.01$, MADRS: $\left.t_{2}=-3.49, p=0.03\right)$. Figure $3 \mathrm{~b}$ illustrates these relationships for both measures and individually for all patients.

Common side effects of depression treatment include agitation, sedation, sleep disturbances, and night sweats. Our patients experienced none of these, and no other negative side effects were observed.

\section{PET Imaging}

Statistically comparing 'before' and 'after' stimulation PET scans shows in which regions of the brain did metabolism increase (seen in Figure 2 as orange/yellow) or decrease (blue) across all patients as a function of the stimulation. We observed significant activations (eg greater metabolism following 1 week of stimulation) in bilateral ventral striatum (including the nucleus accumbens), bilateral dorsolateral and dorsomedial prefrontal cortex and cingulate cortex, and bilateral amygdala. Additionally, we observed deactivations (eg decreased metabolism following 1 week of stimulation) in the ventromedial and ventrolateral prefrontal cortex, dorsal caudate nucleus, and thalamus. In order to present the results, we chose the relatively low significance threshold of $p<0.05$ to see any changes at all in this very small number of patients. See Table 1 for MNI coordinates of all significant changes in metabolism.

Figure 2 additionally displays normalized PET units for each of the three patients in selected brain regions before and after stimulation, demonstrating consistency of the changes in all patients.

\section{DISCUSSION}

Bilateral DBS to the nucleus accumbens had immediate effects on ratings of depression in these very treatment- refractory patients. This was evidenced by both two-way changes in clinical depression ratings and metabolic changes in brain networks associated with depression. This work expands the small but nascent field of the application of DBS for refractory depression by using an hypothesisdriven approach to target a different specific region (Mayberg et al, 2005). We selected the nucleus accumbens as a target region because of its prominent involvement in reward and motivation processing and its anatomical connectivity to limbic and prefrontal regions including Cg25. This system is dysfunctional in patients with major depression (Tremblay et $a l, 2005$ ), and a recent study established that social defeat stress in animals lead to alterations of the mesolimbic dopamine system, and interestingly, the behavioral changes in these animals were reset by chronic application of a selective serotonin reuptake inhibitor (Berton et al, 2006).

Existing antidepressant treatments focus on combinations of psychotherapy and psychopharmacology. Although psychopharmacological treatments are effective for many patients, they are non-specific in that they significantly alter the neurochemistry of many areas of the brain. This leads to notable side effects that often lead to non-compliance (Keller et al, 2002). A more comprehensive conceptualization of depression that inspires specific, targeted neuromodulatory intervention might allow better treatment modalities for depression associated with fewer side effects. Indeed, there were no side effects in any of our DBS patients.

Other brain stimulation techniques exist that use electrical currents or magnetic fields, such as electroconvulsive therapy, repetitive transcranial magnetic stimulation, and vagus nerve stimulation, all of which have antidepressant effects (George et al, 2000; Schlaepfer and Kosel, 2004; Schlaepfer et al, 2003). However, like antidepressants, these methods are also less focused, affect larger areas of the brain not necessarily associated with depression, and induce side effects to various degrees. In contrast, DBS is extremely focused and directly affects only a very small volume of brain tissue.

The effects we describe are acute in all patients. With the onset of stimulation, we observed a significant reduction of ratings of depression, whereas at masked offset ratings of depression increased. Indeed, in patients 2 and 3, this worsening was so bad that the mask had to be broken and they had to be rescued before conclusion of the 4-week masked placebo stimulation period. These two-way changes, observed in masked patients, are a clear indication that the observed results cannot be explained by placebo effects.

Clinical and neurobiological data led to the reintroduction of anhedonia as one of the core symptoms of depression in the DSM-IV in 1994 (Argyropoulos and Nutt, 1997; Rush and Weissenburger, 1994). We hypothesize that our specific treatment modality with a target in the reward system might positively influence this symptom, possibly by restoring normal function of this system. But anhedonia has been implicated with other chronic and debilitating psychiatric disorders such as obsessive-compulsive disorders (Loas, 1996), substance abuse disorders (Wise, 1996), and schizophrenia (Wolf, 2006). These initial observations that DBS to striatal regions indeed might restore dysfunc- 

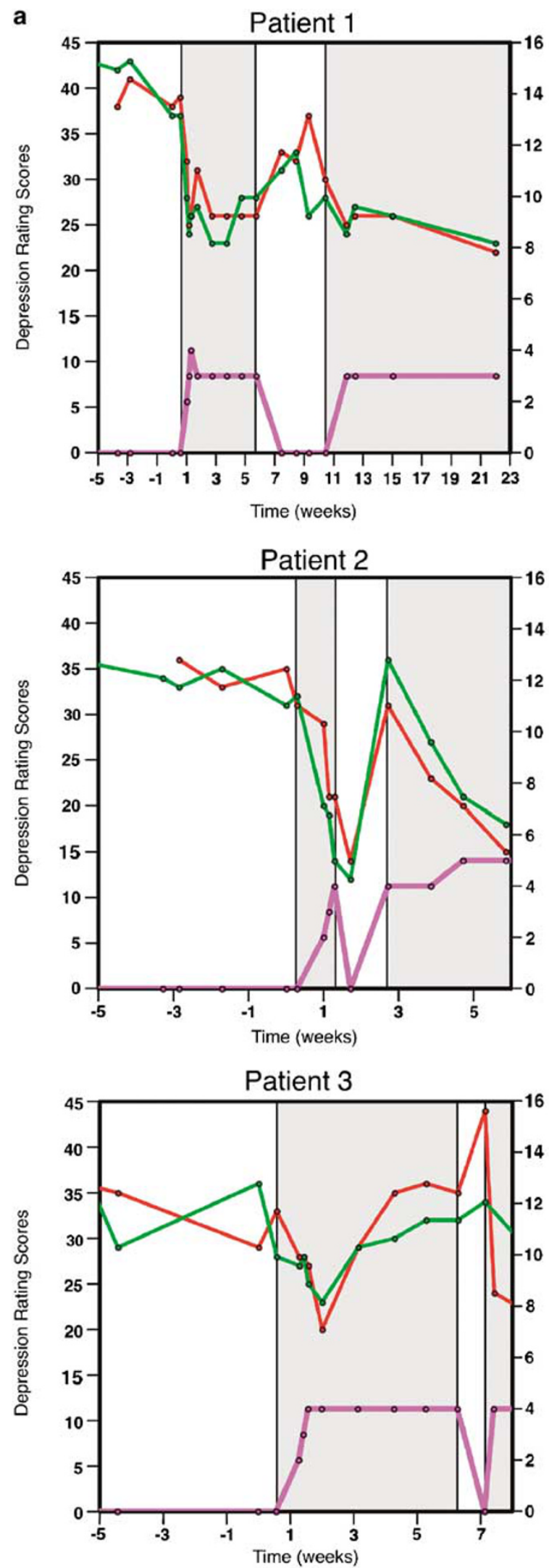

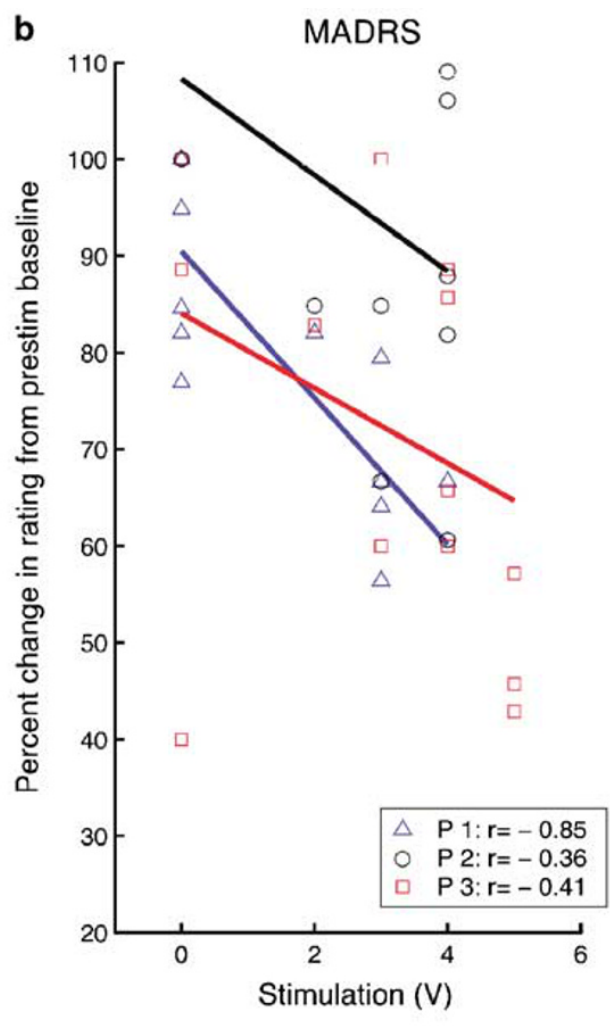

HDRS

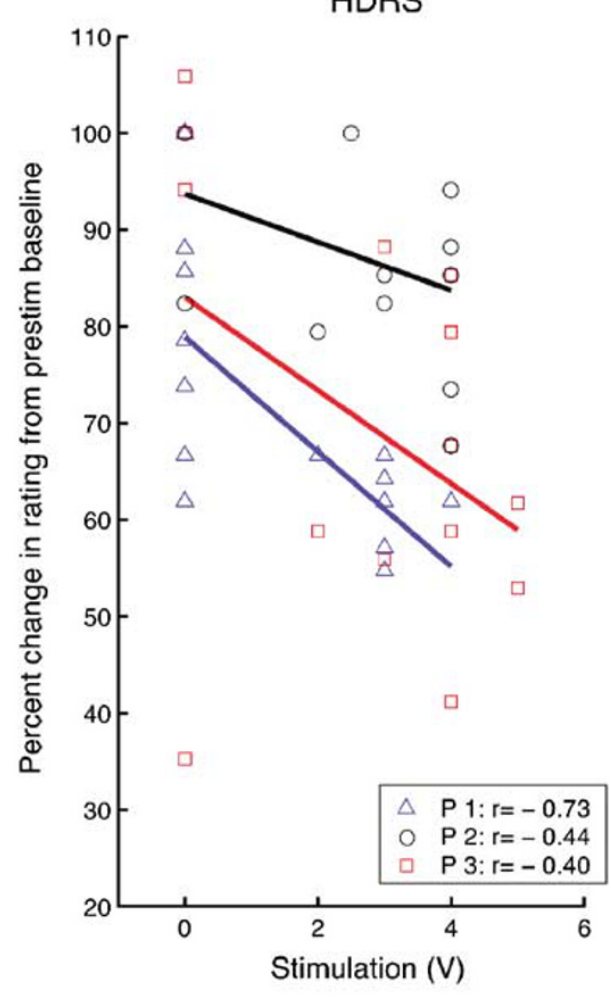

Figure 3 (a) Individual depression score ratings (MADRAS (red) respectively HDRS 24 (green)) and deep brain stimulation amplitude (purple) over time for all patients. Gray shading bars indicate stimulation was on. (b) The effects of stimulation on clinical ratings by correlating ratings of MADRAS and HDRS 24 re-coded as percent change from pre-implantation levels to be able to compare across patients and measures with stimulation parameters. The figures illustrate these relationships for both measures and individually for all patients. 
Table I MNI Coordinates and $t$-Values of Maximally Significant Voxels in the PET Contrast of before Stimulation to after I Week of Constant Accumbens Stimulation

\begin{tabular}{|c|c|c|}
\hline Region & $\mathbf{X}, \mathbf{Y}, \mathbf{Z}(\mathrm{MNI})$ & $t$-value \\
\hline \multicolumn{3}{|l|}{ After $>$ before } \\
\hline L. nucleus accumbens & $10,8,-8$ & 20.90 \\
\hline R. nucleus accumbens & $-10,6,-14$ & 9.41 \\
\hline L. amygdala & $14,-6,-18$ & 20.26 \\
\hline R. amygdala & $18,-2,-18$ & 6.85 \\
\hline R. middle temporal gyrus & $-48,-20,6$ & 61.75 \\
\hline R. middle frontal gyrus & $-52,4,20$ & 11.96 \\
\hline L. middle frontal gyrus & $44,-14,34$ & 19.62 \\
\hline R. anterior hippocampus & $-28,-22,-16$ & 85.36 \\
\hline L. cerebellum & $22,-42,-26$ & 12.60 \\
\hline R. fusiform gyrus & $-42,-50,-8$ & 17.70 \\
\hline L. putamen & $22,-6,-4$ & 8.77 \\
\hline Superior frontal gyrus & $0,-6,64$ & 15.79 \\
\hline R. angular gyrus & $-42,-82,20$ & 7.49 \\
\hline \multicolumn{3}{|l|}{ Before $>$ after } \\
\hline R. lateral orbital gyrus & $-28,40,-12$ & -50.89 \\
\hline L. anterior cingulate & $12,50,0$ & -26.64 \\
\hline Superior frontal gyrus & $-2,64,8$ & -85.36 \\
\hline R. substantia nigra & $-6,-22,-24$ & -43.87 \\
\hline R. inferior frontal gyrus & $-36,42,-4$ & -22.81 \\
\hline L. anterior insula & $38,14,4$ & -27.92 \\
\hline R. thalamus & $-4,-6,8$ & -9.41 \\
\hline L. caudate & $16,20,6$ & -15.79 \\
\hline R. inferior temporal gyrus & $-46,-42,-28$ & -20.26 \\
\hline R. supramarginal gyrus & $-58,-40,38$ & $-|4.5|$ \\
\hline
\end{tabular}

tional processing of reward stimuli lays ground to researching similar approaches for the treatment of those other disorders.

The reported behavioral changes that occurred in these patients beg the question of what neurobiological changes occurred that underlie these behavioral and clinical shifts. Depression is associated with pathological and abnormal functioning of brain regions including striatum and prefrontal cortex. We speculated that DBS to the nucleus accumbens, through its anatomical and functional connections with other limbic and prefrontal structures, would restore activity in these regions to levels seen in nondepressed individuals. This hypothesis is supported by our PET data. One week of DBS significantly increased metabolism in the nucleus accumbens, amygdala, and dorsolateral and dorsomedial prefrontal cortex, and decreased metabolism in the ventral and ventrolateral medial prefrontal cortex. These latter regions have been described to be hypermetabolic in depressed states. In patients with obsessive-compulsive disorder, selective monopolar stimulation of essentially the same target - albeit with a larger single electrode contact - produced acute metabolic effects after 10-20 min that are generally consistent with those found in our study after 1 week of stimulation, supporting the hypothesis that cortical-thalamic-striatal-cortical circuitry can be modulated by DBS at this target site (Rauch et al, 2006). The group of Mayberg et al (2005) has demonstrated that improvement of depressive symptomatology in response to different treatment modalities including DBS to it is invariably associated with a metabolic decrease in Brodmann area 25 (Cg25). In our preliminary PET analysis we did not observe significant decreases in Cg25, despite the fact that there are dense monosynaptic projections from this region to the nucleus accumbens. However, it might well be that these decreases in $\operatorname{Cg} 25$ might take longer to develop than the short interval of only 1 week between initiation of stimulation and the PET study.

\section{CONCLUSIONS AND LIMITATIONS}

These findings should be taken as very preliminary at the clinical level. We certainly do not suggest that DBS to the nucleus accumbens is a 'magic bullet' procedure that will cure depression. We and other groups must still demonstrate clinical usefulness of different DBS approaches. However, it is important to note that the patients in this study had severe and long-lasting depression that was not helped by traditional methods. Although DBS to the nucleus accumbens did not 'cure' their depression, it remains the only treatment option so far that has minimized levels of depression in many years and many different treatment attempts. This is particularly important because we did not observe any neurological or psychological side effects of this treatment.

Taken together, using a translational approach spanning basic and clinical neuroscience, we found that DBS to brain structures mediating reward and motivation processes has significant, two-way, acute clinical effects on depressive symptomatology. These findings might offer a focused and novel approach to treating refractory major depression with a favorable efficacy to side effect profile.

\section{ACKNOWLEDGEMENTS}

We thankful patients and their families described in this study for participating not only in the clinical part of this study but also in numerous scientific protocols.

Contributions: TS and VS were the study coordinators; TS, VS, and MKo were involved in the protocol design; CF, $\mathrm{DB}, \mathrm{NA}$, and $\mathrm{MKr}$ were involved in the collection and analysis of data; MC and TS were involved in the statistical analysis, and writing and elaboration of the initial paper. AJ was involved in the analysis of the PET data. DL was involved in the surgical procedures and assessment of patients. All authors reviewed and revised the paper.

\section{CONFLICTS OF INTEREST}

This study is funded in part by an investigator-initiated grant by Medtronic Inc. to Drs Schlaepfer and Sturm. The sponsor had no influence whatsoever on planning, analysis, and drafting of the article, the article has not been seen by any employee of the sponsor at the time of submission or revision. 


\section{REFERENCES}

Abelson JL, Curtis GC, Sagher O, Albucher RC, Harrigan M, Taylor SF et al (2005). Deep brain stimulation for refractory obsessivecompulsive disorder. Biol Psychiatry 57: 510-516.

Adinoff B (2004). Neurobiologic processes in drug reward and addiction. Harv Rev Psychiatry 12: 305-320.

Aharon I, Etcoff N, Ariely D, Chabris CF, O'Connor E, Breiter HC (2001). Beautiful faces have variable reward value: fMRI and behavioral evidence. Neuron 32: 537-551.

Andrews JM, Nemeroff CB (1994). Contemporary management of depression. Am J Med 97: 24S-32S.

Argyropoulos SV, Nutt DJ (1997). Anhedonia and chronic mild stress model in depression. Psychopharmacology (Berlin) 134: 333-336, discussion 371-337.

Auer DP, Putz B, Kraft E, Lipinski B, Schill J, Holsboer F (2000). Reduced glutamate in the anterior cingulate cortex in depression: an in vivo proton magnetic resonance spectroscopy study. Biol Psychiatry 47: 305-313.

Bartenstein P, Asenbaum S, Catafau A, Halldin C, Pilowski L, Pupi A et al (2002). European Association of Nuclear Medicine procedure guidelines for brain imaging using [(18)F]FDG. Eur J Nucl Med Mol Imaging 29: BP43-BP48.

Berton O, McClung CA, Dileone RJ, Krishnan V, Renthal W, Russo $\mathrm{SJ}$ et al (2006). Essential role of BDNF in the mesolimbic dopamine pathway in social defeat stress. Science 311: 864-868.

Blood AJ, Zatorre RJ (2001). Intensely pleasurable responses to music correlate with activity in brain regions implicated in reward and emotion. Proc Natl Acad Sci USA 98: 11818-11823.

Breiter HC, Gollub RL, Weisskoff RM, Kennedy DN, Makris N, Berke JD et al (1997). Acute effects of cocaine on human brain activity and emotion. Neuron 19: 591-611.

Cohen MX, Young J, Baek JM, Kessler C, Ranganath C (2005). Individual differences in extraversion and dopamine genetics predict neural reward responses. Brain Res Cogn Brain Res 25: 851-861.

de la Fuente-Fernandez R, Phillips AG, Zamburlini M, Sossi V, Calne DB, Ruth TJ et al (2002). Dopamine release in human ventral striatum and expectation of reward. Behav Brain Res 136: 359-363.

Deuschl G, Bain P (2002). Deep brain stimulation for tremor: patient selection and evaluation. Mov Disord 17(Suppl 3): S102S111.

Deuschl G, Schade-Brittinger C, Krack P, Volkmann J, Schafer H, Botzel $\mathrm{K}$ et al (2006). A randomized trial of deep-brain stimulation for Parkinson's disease. $N$ Engl J Med 355: 896-908.

Doyon WM, Anders SK, Ramachandra VS, Czachowski CL, Gonzales RA (2005). Effect of operant self-administration of $10 \%$ ethanol plus $10 \%$ sucrose on dopamine and ethanol concentrations in the nucleus accumbens. J Neurochem 93: 1469-1481.

Drevets WC, Gautier C, Price JC, Kupfer DJ, Kinahan PE, Grace AA et al (2001). Amphetamine-induced dopamine release in human ventral striatum correlates with euphoria. Biol Psychiatry 49: 81-96.

Flaherty AW, Williams ZM, Amirnovin R, Kasper E, Rauch SL, Cosgrove GR et al (2005). Deep brain stimulation of the anterior internal capsule for the treatment of Tourette syndrome: technical case report. Neurosurgery 57: E403, discussion E403.

George MS, Sackeim HA, Rush AJ, Marangell LB, Nahas Z, Husain MM et al (2000). Vagus nerve stimulation: a new tool for brain research and therapy. Biol Psychiatry 47: 287-295.

Ghika J, Villemure JG, Fankhauser H, Favre J, Assal G, Ghika-Schmid F (1998). Efficiency and safety of bilateral contemporaneous pallidal stimulation (deep brain stimulation) in levodopa-responsive patients with Parkinson's disease with severe motor fluctuations: a 2-year follow-up review. J Neurosurg 89: 713-718.

Greenberg BD, Price LH, Rauch SL, Friehs G, Noren G, Malone D et al (2003). Neurosurgery for intractable obsessive-compulsive disorder and depression: critical issues. Neurosurg Clin N Am 14: 199-212.

Greenberg BD, Rezai AR (2003). Mechanisms and the current state of deep brain stimulation in neuropsychiatry. CNS Spectr 8: 522-526.

Gross RE (2004). Deep brain stimulation in the treatment of neurological and psychiatric disease. Expert Rev Neurother 4: 465-478.

Hamilton M (1960). Rating scale for depression. J Neurol Neurosurg Psychiatry 23: 56-61.

Heath RG (1972). Pleasure and brain activity in man. Deep and surface electroencephalograms during orgasm. J Nerv Ment Dis 154: 3-18.

Houeto JL, Karachi C, Mallet L, Pillon B, Yelnik J, Mesnage V et al (2005). Tourette's syndrome and deep brain stimulation. J Neurol Neurosurg Psychiatry 76: 992-995.

Ito R, Robbins TW, Everitt BJ (2004). Differential control over cocaine-seeking behavior by nucleus accumbens core and shell. Nat Neurosci 7: 389-397.

Jasinski D, Johnson R, Henningfield J (1984). Abuse liability assessment in human subjects. Trends Pharmacol Sci 5: 196-200.

Jones DL, Mogenson GJ (1980). Nucleus accumbens to globus pallidus GABA projection: electrophysiological and iontophoretic investigations. Brain Res 188: 93-105.

Juckel G, Schlagenhauf F, Koslowski M, Wustenberg T, Villringer A, Knutson B et al (2006). Dysfunction of ventral striatal reward prediction in schizophrenia. Neuroimage 29: 409-416.

Keller MB, Hirschfeld RM, Demyttenaere K, Baldwin DS (2002). Optimizing outcomes in depression: focus on antidepressant compliance. Int Clin Psychopharmacol 17: 265-271.

Keller MB, Lavori PW, Mueller TI, Endicott J, Coryell W, Hirschfeld HM et al (1992). Time to recovery, chronicity, and levels of psychopathology in major depression. A 5-year prospective follow-up of 431 subjects. Arch Gen Psychiatry 49: 809-816.

Kelley AE, Stinus L (1984). The distribution of the projection from the parataenial nucleus of the thalamus to the nucleus accumbens in the rat: an autoradiographic study. Exp Brain Res 54: 499-512.

Knutson B, Adams CM, Fong GW, Hommer D (2001a). Anticipation of increasing monetary reward selectively recruits nucleus accumbens. J Neurosci 21: RC159.

Knutson B, Fong GW, Adams CM, Varner JL, Hommer D (2001b). Dissociation of reward anticipation and outcome with eventrelated fMRI. Neuroreport 12: 3683-3687.

Knutson B, Fong GW, Bennett SM, Adams CM, Hommer D (2003). A region of mesial prefrontal cortex tracks monetarily rewarding outcomes: characterization with rapid event-related fMRI. Neuroimage 18: 263-272.

Kornetsky C (2004). Brain-stimulation reward, morphine-induced oral stereotypy, and sensitization: implications for abuse. Neurosci Biobehav Rev 27: 777-786.

Loas G (1996). Vulnerability to depression: a model centered on anhedonia. J Affect Disord 41: 39-53.

Mann JJ (2005). The medical management of depression. $N$ Engl J Med 353: 1819-1834.

Mayberg H (2002). Modulating limbic-cortical circuits in depression: targets of antidepressant treatments. Semin Clin Neuropsychiatry 7: 255-268.

Mayberg HS (1997). Limbic-cortical dysregulation: a proposed model of depression. J Neuropsychiatry Clin Neurosci 9: 471-481.

Mayberg HS, Liotti M, Brannan SK, McGinnis S, Mahurin RK, Jerabek PA et al (1999). Reciprocal limbic-cortical function and negative mood: converging PET findings in depression and normal sadness. Am J Psychiatry 156: 675-682. 
Mayberg HS, Lozano AM, Voon V, McNeely HE, Seminowicz D, Hamani C et al (2005). Deep brain stimulation for treatmentresistant depression. Neuron 45: 651-660.

McClelland III S, Ford B, Senatus PB, Winfield LM, Du YE, Pullman SL et al (2005). Subthalamic stimulation for Parkinson disease: determination of electrode location necessary for clinical efficacy. Neurosurg Focus 19: E12.

Mirza Y, Tang J, Russell A, Banerjee SP, Bhandari R, Ivey J et al (2004). Reduced anterior cingulate cortex glutamatergic concentrations in childhood major depression. J Am Acad Child Adolesc Psychiatry 43: 341-348.

Mogenson GJ, Swanson LW, Wu M (1983). Neural projections from nucleus accumbens to globus pallidus, substantia innominata, and lateral preoptic-lateral hypothalamic area: an anatomical and electrophysiological investigation in the rat. J Neurosci 3: 189-202.

Montgomery SA, Asberg M (1979). A new depression scale designed to be sensitive to change. Br J Psychiatry 134: 382-389.

Nauta WJ, Domesick VB (1984). Afferent and efferent relationships of the basal ganglia. Ciba Found Symp 107: 3-29.

O'Doherty J, Kringelbach ML, Rolls ET, Hornak J, Andrews C (2001). Abstract reward and punishment representations in the human orbitofrontal cortex. Nat Neurosci 4: 95-102.

Rauch SL, Dougherty DD, Malone D, Rezai A, Friehs G, Fischman $\mathrm{AJ}$ et al (2006). A functional neuroimaging investigation of deep brain stimulation in patients with obsessive-compulsive disorder. J Neurosurg 104: 558-565.

Rush AJ, Weissenburger JE (1994). Melancholic symptom features and DSM-IV. Am J Psychiatry 151: 489-498.
Schlaepfer T, Kosel M (2004). Novel physical treatments for major depression: vagus nerve stimulation, transcranial magnetic stimulation and magnetic seizure therapy. Curr Opin Psychiatry 17: 15-20.

Schlaepfer TE, Kosel M, Nemeroff CB (2003). Efficacy of repetitive Transcranial Magnetic Stimulation (rTMS) in the treatment of affective disorders. Neuropsychopharmacology 28: 201-205.

Schlaepfer TE, Lieb K (2005). Deep brain stimulation for treatment refractory depression. Lancet 366: 1420-1422.

Schultz W (2004). Neural coding of basic reward terms of animal learning theory, game theory, microeconomics and behavioural ecology. Curr Opin Neurobiol 14: 139-147.

Seminowicz DA, Mayberg HS, McIntosh AR, Goldapple K, Kennedy S, Segal Z et al (2004). Limbic-frontal circuitry in major depression: a path modeling metanalysis. Neuroimage 22: 409-418.

Sturm V, Lenartz D, Koulousakis A, Treuer H, Herholz K, Klein JC et al (2003). The nucleus accumbens: a target for deep brain stimulation in obsessive-compulsive- and anxiety-disorders. J Chem Neuroanat 26: 293-299.

Tremblay LK, Naranjo CA, Graham SJ, Herrmann N, Mayberg HS, Hevenor S et al (2005). Functional neuroanatomical substrates of altered reward processing in major depressive disorder revealed by a dopaminergic probe. Arch Gen Psychiatry 62: $1228-1236$

Wise RA (1996). Addictive drugs and brain stimulation reward. Annu Rev Neurosci 19: 319-340.

Wolf DH (2006). Anhedonia in schizophrenia. Current psychiatry reports 8: $322-328$. 